\title{
Calculating the Entropy Loss on Adsorption of Organic Molecules at Insulating Surfaces
}

\author{
Julian Gaberle, ${ }^{* \dagger}$ David Z. Gao, ${ }^{*} \dagger$ Matthew B. Watkins, ${ }^{\ddagger}$ and Alexander L. \\ Shluger \\ $\dagger$ Department of Physics and Astronomy, University College London, Gower Street, London \\ WC1E 6BT, United Kingdom \\ $\ddagger$ School of Maths and Physics, University of Lincoln, Brayford Pool, Lincoln LN6 7TS, \\ United Kingdom \\ ฯWPI-AIMR, Tohoku University, 2-1-1 Katahira, Aoba-ku, Sendai, 980-8577, Japan \\ E-mail: julian.gaberle.10@ucl.ac.uk; david.gao.10@ucl.ac.uk \\ Phone: $+44.79 .5153 .7952 ;++1.831 .233 .3329$
}




\begin{abstract}
Although it is recognized that dynamic behavior of adsorbing molecules strongly affects the entropic contribution to adsorption free energy, detailed studies of the adsorption entropy of large organic molecules at insulating surfaces are still rare. We compared adsorption of two different functionalized organic molecules, 1,3,5-tri-(4-cyano4,4 biphenyl)-benzene (TCB) and 1,4-bis(cyanophenyl)-2,5-bis(decyloxy)benzene (CDB), on the $\mathrm{KCl}$ (001) surface using density functional theory (DFT) and molecular dynamics (MD) simulations. The accuracy of the van der Waals corrected DFT-D3 was benchmarked using Møller-Plesset perturbation theory calculations. Classical force fields were then parameterized for both the TCB and CDB molecules on the $\mathrm{KCl}(001)$ surface. These force fields were used to perform potential of mean force (PMF) calculations of adsorption of individual molecules and extract information on the entropic contributions to adsorption energy. The results demonstrate that entropy losses upon adsorption are significant for flexible molecules, such as considered here, and even at relatively low temperatures (e.g. $400 \mathrm{~K}$ ) can match the enthalpy contribution to adsorption energy.
\end{abstract}

\title{
Introduction
}

Understanding the adsorption of organic molecules at insulating substrates at room and elevated temperatures is important in many fields, such as sensor technologies, ${ }^{1,2}$ coatings, ${ }^{3}$ catalysis, ${ }^{4}$ functional films ${ }^{5}$ and molecular electronics. ${ }^{6-8}$ Designing substrates and molecules targeting these applications requires deep understanding of molecular interactions to achieve control over the morphology of these films. ${ }^{2,9}$ Often these applications rely on self-assembled films with self-healing properties. It has been shown that formation of such films can be achieved by carefully tuning the balance between the entropy of adsorption and energy of molecule-molecule and molecule-substrate interactions within a physisorbed layer. ${ }^{10-15}$

Adsorption energies and geometries of functionalized molecules have been successfully 

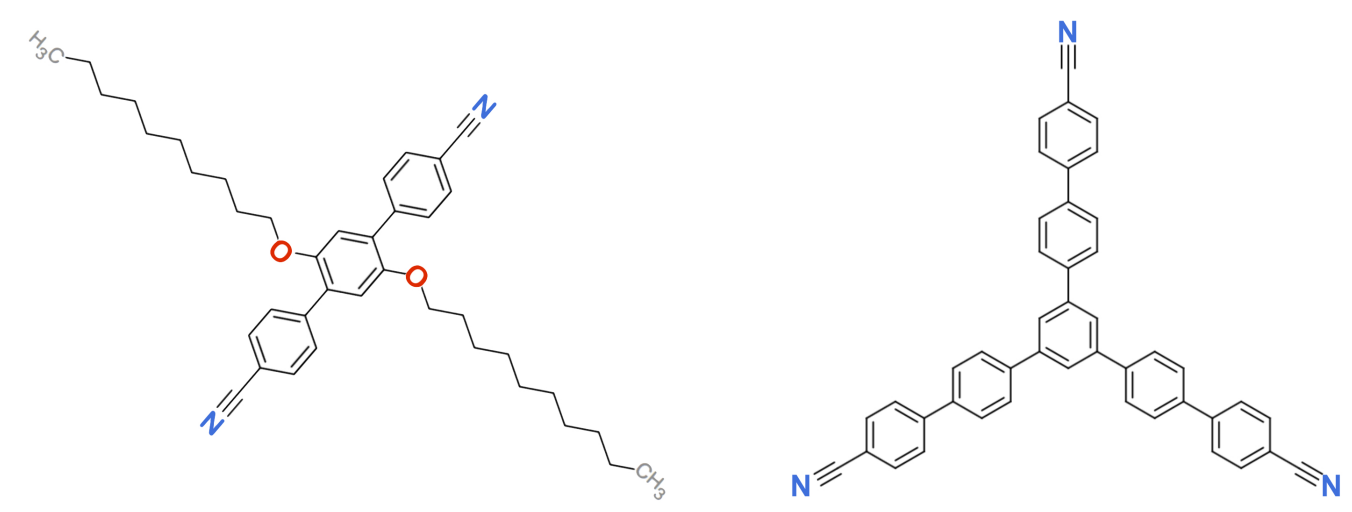

Figure 1: Chemical structure of CDB (1,4-bis(cyanophenyl)-2,5-bis(decyloxy)benzene) on the left and TCB (1,3,5-tri-(4-cyano-4,4 biphenyl)-benzene) molecule on the right.

characterized in the past using various experimental techniques. ${ }^{16-24}$ Adsorption geometries in particular can be investigated using scanning probe methods such as non-contact atomic force microscopy (NC-AFM). ${ }^{25-27}$ However, achieving high resolution in NC-AFM images at room temperature is still a challenge ${ }^{28}$ and often the interpretation of images down to an atomistic level requires a detailed understanding of the tip-sample interactions. ${ }^{29-31}$ Theoretical calculations can provide a better understanding of adsorption geometries, film structures and nucleation sites. Previous work on molecular adsorption on insulators using DFT has been often restricted to ground state calculations that represent a frozen $0 \mathrm{~K}$ system. ${ }^{12,32}$ Since real experiments are performed at higher temperatures, the entropic contributions to adsorption and dynamic properties of the adsorbing molecules may have a qualitative effect on molecular behavior. Although the importance of entropic contribution to the free energy of adsorption has been long recognized (see e.g. ref. ${ }^{33}$ ), detailed studies started to appear more recently in the context of molecular association in solutions, ${ }^{34}$ molecular adsorption from solutions,${ }^{35}$ formation of ordered monolayers at surfaces, ${ }^{15}$ and understanding temperature programmed desorption (TPD) experiments. ${ }^{36}$ The experimentally measured enthalpies and entropies of adsorption of relatively small molecules on oxide surfaces have been summarized in ref. ${ }^{37}$ However, detailed studies of the adsorption of large organic molecules at insulating surfaces are still challenging and often confined to static simulations. ${ }^{12,32,38}$

Our long-term aim is to design molecules that can form stable functional supramolecular 
structures on bulk insulators at room temperature. In the previous work ${ }^{39}$ we have characterized the adsorption of individual 1,4-bis(cyanophenyl)-2,5-bis(decyloxy)benzene (CDB) molecules on the inert $\mathrm{KCl}$ (001) surface and discussed how these flexible molecules (see Figure 1) form highly ordered, 2D, porous molecular domains. In contrast, the 1,3,5-tri-(4cyano-4,4 biphenyl)-benzene (TCB) molecule does not possess flexible hydrocarbon chains, has a much more rigid frame (see Figure 1) but can still rotate easily on the KCl surface. These contrasting features present an excellent opportunity to look in more detail at the effects of flexibility of molecular structure and the ensuing entropy loss in adsorption of organic molecules on insulating surfaces. In this paper, we compare the adsorption of individual $\mathrm{CDB}$ and $\mathrm{TCB}$ molecules on $\mathrm{KCl}(001)$ whereas in future work we will discuss the effect of their structure on the nucleation and growth of monolayer structures on $\mathrm{KCl}(001)$.

Due to the large size of these molecules, these simulations are computationally expensive and limit what can be achieved using ab initio methods. Therefore classical force fields were parameterized for these two organic molecules using genetic algorithm methods. ${ }^{40} \mathrm{We}$ benchmarked van der Waals (vdW)-corrected PBE-D3 Density Functional Theory (DFT) calculations against high accuracy Møller-Plesset perturbation theory (MP2) for a several representative molecular fragments adsorbed on $\mathrm{KCl}(001)$. This allowed us to probe the entropic contributions to adsorption and examine the dynamic properties of molecular adsorption using MD simulations and PMF calculations.

\section{Methods of Calculations}

The calculations were performed using a wide range of techniques, including density functional theory (DFT), hybrid quantum mechanics/ molecular mechanics (QM/MM), ${ }^{41,42}$ second order Møller-Plesset perturbation theory (MP2), and classical molecular dynamics (MD). DFT was used to study the adsorption geometries and electronic structure of TCB molecules adsorbed onto $\mathrm{KCl}$ (001). However, DFT calculations on systems of this size are too com- 
putationally expensive to probe their dynamic properties. QM/MM techniques were used to reduce the cost of performing ab initio calculations and facilitated the generation of an extensive dataset of configurations. This data set was then used to parametrize a classical force field for the system in order to run classical MD. Since vdW interactions are not well represented within DFT, we compared our electronic structure data to MP2 results on small molecular fragments. Finally, potential of mean force calculations were used to calculate the adsorption energies at elevated temperatures and to evaluate the entropic contribution to adsorption energy.

\section{Density Functional Theory}

DFT calculations were performed using the CP2K code with the Gaussian plane wave (GPW) method $^{43}$ and the PBE GGA local density functional. ${ }^{44}$ We employed semi-empirical longrange dispersion corrections ${ }^{45}$ in order to represent vdW interactions in the system. Finally, the MOLOPT ${ }^{46}$ basis set was selected to minimize basis set superposition error and a plane wave cutoff of $400 \mathrm{Ry}$ was used. The $\mathrm{KCl}$ surface was represented using four atomic layers with one fixed layer at the bottom and three layers of relaxed atoms. These calculations produce a band gap of $5.4 \mathrm{eV}$, surface rumpling of $0.3 \AA$, and a lattice constant of $6.3 \AA$ in agreement with experimental values. ${ }^{47}$

\section{Second Order Møller-Plesset Perturbation Theory}

We used the implementation of MP2 within the CP2K package. ${ }^{48,49}$ Series of correlation consistent basis sets of double, treble and quadruple zeta quality for elements $\mathrm{C}, \mathrm{H}, \mathrm{N}$, O were selected, ${ }^{50}$ which are the Dunning cc-pVXZ basis sets adapted to be compatible with the GTH pseudo potentials employed. ${ }^{50-52}$ We optimized basis sets using the same methodology for $\mathrm{Cl}$ and $\mathrm{K}$. In the case of $\mathrm{K}$ we had to optimize the correlation functions as well, since this element does not have a Dunning basis set published. This was achieved by optimizing the energies of a $\mathrm{K}$ atom using a gradient-less optimizer at the MP2 level with the 
standard hierarchy of correlation consistent functions. The resolution of the identity (RI) approximation $^{53,54}$ was used - RI basis sets for $\mathrm{K}$ and $\mathrm{Cl}$ were generated using an internal procedure in $\mathrm{CP} 2 \mathrm{~K}$ and were confirmed to give transferable errors of $10 \mu \mathrm{Ha}$ per atom, consistent with the quality of the RI basis sets for $\mathrm{H}, \mathrm{C}, \mathrm{N}, \mathrm{O}$, on a succession of bulk $\mathrm{KCl}$ supercells.

The $\mathrm{KCl}$ bulk lattice constant optimized at the MP2 level ${ }^{49}$ is $6.37 \AA$. Geometry optimizations were performed until forces on relaxing atoms were smaller than $0.025 \mathrm{eV} / \mathrm{A}$. The bottom layer of the 3 layer thick, 108 atom $\mathrm{KCl}$ slab was fixed in its bulk position. Adsorption energies were found by extrapolation to the basis set limit using the approximate formulae of Truhlar et. al.: ${ }^{55}$

$$
E_{X}^{n}=E_{X \rightarrow \infty}^{n \rightarrow \infty}+A X^{-3}
$$

where $n$ represents the highest angular momentum in an augmented correlation-consistent basis set, $\mathrm{X}=2,3$, and 4 for the cc-DZVP, cc-TZVP, and cc-QZVP basis sets, respectively. We confirmed that the raw numbers and BSSE corrected results extrapolated to the same number within $0.025 \mathrm{eV}$. The optimized basis sets and example CP2K input files are provided as supplementary information.

\section{Fitting Classical Potentials}

As experimental data on the adsorption energies of TCB and CDB molecules on $\mathrm{KCl}(001)$ are not available, we used a previously described method ${ }^{40}$ to efficiently generate classical force fields for the molecule-surface interactions. The inter- and intra-molecular interactions were

represented by the $\mathrm{CHARMM}^{56}$ force field and the surface was represented by a Buckingham potential as parameterized by Catlow et al. ${ }^{57}$ The force field for $\mathrm{CDB}$ molecules on $\mathrm{KCl}(001)$ is provided in a previous publication. ${ }^{40}$

A hybrid QM/MM method ${ }^{58,59}$ was used to increase the surface area in these simulations 
and reduce the computational cost so that a fitting library of geometries and forces could be produced. The $\mathrm{KCl}$ (001) surface was represented using one layer of atoms treated quantum mechanically and three layers of atoms treated classically. The QM region was treated using the DFT method, basis sets, dispersion corrections, and cutoffs described above. The MM region was represented using a set of classical parameters by Catlow et al. ${ }^{57}$ and the charges on ions were smeared using Gaussian functions based on the ionic radii of the atoms. Using the QM/MM method we produced a band gap of $4.6 \mathrm{eV}$ for the $\mathrm{KCl}(001)$ surface, surface rumpling of $0.3 \AA$, and a lattice constant of $6.3 \AA$. A more detailed description and analysis of this method can be found in a previous publication. ${ }^{39}$

The training set created for $\mathrm{TCB}$ on $\mathrm{KCl}(001)$ consisted of 240 configurations. The first 80 frames (or configurations) were obtained from QM/MM MD calculations starting from the ground state of the adsorbed molecule. The starting position of the molecule was then rotated in $15^{\circ}$ increments around the surface normal and a new trajectory was calculated at each starting position, resulting in 120 additional frames. The final 40 frames were created by artificially positioning the molecule at varying heights above the surface. These frames represent situations that would not normally be probed via room temperature MD and provide information about the system when the molecule is farther or closer to the surface plane.

Within the genetic algorithm scheme, we selected a population of 1024 elements which was then evolved over 1000 generations. In each generation, $5 \%$ of the total population was randomized, duplicate members were randomized, and each element had a $0.2 \%$ chance of mutation in order to reduce artificial convergence. The Morse potential form was chosen for consistency with the previous work on the CDB molecule. ${ }^{40}$

\section{Potential of Mean Force}

Potential of mean force calculations give insight into how the free energy of a system changes as a function of atomic coordinates. ${ }^{34,60}$ The free energy difference between two states along 
a reaction coordinate $\xi$, which parameterises the Hamiltonian $\mathrm{H}$, is defined as

$$
W\left(\xi_{2}\right)-W\left(\xi_{1}\right)=\int_{\xi_{1}}^{\xi_{2}} d \xi^{\prime}\left\langle\frac{\delta H}{\delta \xi}\right\rangle_{\xi^{\prime}}
$$

where $\langle\ldots\rangle_{\xi}$ is a statistical average over the equilibrium ensemble corresponding to parameter value $\xi^{\prime}$ with the condition $\xi(\mathbf{r})=\xi^{\prime} \cdot{ }^{61}$ Minus the integrand is often termed the mean force and hence the free energy is the potential of mean force.

Potential of mean force calculations were done by performing molecular dynamics simulations in the (NVT) ensemble with a Nosé-Hoover thermostat to control the temperature of the system and using the velocity Verlet integrator to ensure positions and velocities are updated during the same time step. Each MD run was 3.0 ns long with a timestep of 1.0 fs. The reaction path is defined parallel to the normal of the surface plane. MD runs were set up by placing a single $\mathrm{TCB}$ molecule on a $\mathrm{KCl}$ substrate, which was represented using a four layer slab composed of 1024 atoms. To prevent the substrate from drifting the bottom layer of the four substrate layers was frozen in its bulk position. During these MD runs the $z$ - coordinate (normal to the plane of the surface) of the molecule's center of mass (COM) was fixed. There were no additional constraints made to the molecule to ensure that the molecule could rotate, vibrate and translate in the plane parallel to the surface at distance $z$ in order to sample the ensemble phase space. In order to obtain a reliable potential, the molecule was placed at 76 distances in the range of $3.0 \AA$ to $21.0 \AA$ from the surface with the finest sampling close to the surface. From standard classical mechanics the potential $\phi_{z}$ normal to the surface is defined as:

$$
d \phi_{z} / d z=-\left\langle F_{z}\right\rangle_{z}
$$

where $\left\langle F_{z}\right\rangle$ is the average force normal to the surface between the substrate and the molecule. ${ }^{62}$ Thus the potential can be easily calculated from the average force $\left\langle F_{z}\right\rangle$ by integration with respect to the reaction coordinate $z$. The first 2.0 ps of each MD run were used 
to equilibrate the system and thus were not included in the average. Sample input scripts and parameters used are provided in the supplementary information.

\section{Results and Discussion}

\section{vdW Contributions to Adsorption energy}

Initial DFT calculations of TCB and CDB molecules adsorbed onto $\mathrm{KCl}$ (001) were performed to determine the adsorption energy and geometry. However, results indicated that vdW interactions account for a large portion of the adsorption enthalpy. The adsorption enthalpy of a TCB molecule on the $\mathrm{KCl}$ (001) surface was calculated to be $4.3 \mathrm{eV}$ with a $3.3 \mathrm{eV}$ contribution from long range dispersion corrections using the DFT-D3 method. Similarly, the adsorption enthalpy of $\mathrm{CDB}$ on $\mathrm{KCl}(001)$ was calculated to be $3.1 \mathrm{eV}$ with a $2.4 \mathrm{eV}$ dispersion contribution. To benchmark the accuracy of semi-empirical vdW corrections we compare our results to MP2 calculations performed using the CP2K code. These methods give a good, if normally slightly overestimated, description of vdW interactions.

Since the TCB and CDB molecules are too large to treat using such computationally expensive methods, we chose several smaller molecules representative of molecular fragments to treat on the $\mathrm{KCl}$ (001) surface. These included the propane molecule, a benzene ring, and a benzene ring functionalized by a cyano-group. The results of these calculations are presented in Table 1.

Table 1: Comparison of the adsorption energies for several molecules on the $\mathrm{KCl}$ (001) surface calculated using MP2 and DFT PBE-D3.

\begin{tabular}{|c|cc|}
\hline Adsorption Energy on $\mathrm{KCl}(001)$ & MP2 & DFT PBE-D3 \\
\hline \hline Propane & $0.15 \mathrm{eV}$ & $0.18 \mathrm{eV}$ \\
Benzene & $0.37 \mathrm{eV}$ & $0.45 \mathrm{eV}$ \\
CN-Benzene & $0.41 \mathrm{eV}$ & $0.70 \mathrm{eV}$ \\
\hline
\end{tabular}

These results show that for the propane and benzene molecules, DFT PBE-D3 does provide a good description of the adsorption energy. However, the functionalized CN-benzene 
adsorption energy is overestimated. This discrepancy can be explained by considering how vdW is treated within PBE-D3. Since the dispersion correction is accounted for using a pairwise additive scheme, it cannot account for the electron withdrawing CN functional group. It has indeed been previously proposed that the presence of such withdrawing groups lowers the adsorption energy of benzene rings. ${ }^{63}$ Assuming the additive character of vdW interaction and using these results, we would expect the adsorption energy of TCB and CDB molecules to be overestimated by about 20\% using DFT PBE-D3.

\section{Evaluating the Force-Fields}

After benchmarking DFT-D3 data against MP2 results, we parameterized classical force fields for the TCB molecule adsorbing onto $\mathrm{KCl}(001)$ using genetic algorithm methods. These force fields were then evaluated by comparing adsorption energy and geometries with the original DFT results. Energy minimization calculations were performed in order to find the lowest energy adsorption geometry. A single TCB molecule was systematically placed on the surface with its centre of mass at different sites of the unit cell. At each of these configurations the molecule was rotated by $2^{\circ}$ increments around the normal of the surface plane. The surface slab consisted of four layers of $\mathrm{KCl}$ with the bottom layer frozen in order to prevent drifting within the simulation box. A $80 \AA$ vacuum distance was used between periodically translated slabs.

Two stable configurations found in the classical simulations are shown in Figure 2. In both configurations the cyano-groups (CN groups) are located nearest to a $\mathrm{K}+$ site. The configuration in Figure 2(a) exhibits mirror symmetry along the $\langle 110\rangle$ direction and one leg of the molecule aligns with a cation row. This is energetically favorable with an adsorption enthalpy of $4.55 \mathrm{eV}$. While in vacuum the benzene rings of the legs form a torsion angle of $31^{\circ}$, the leg above the surface cation row is very flat (torsion angle of $0.6^{\circ}$ between the two benzene rings) and the two benzene rings of the other two legs rotate to form a $4^{\circ}$ angle with each other. The adsorption energies compare within a $7 \%$ deviation with the data obtained 

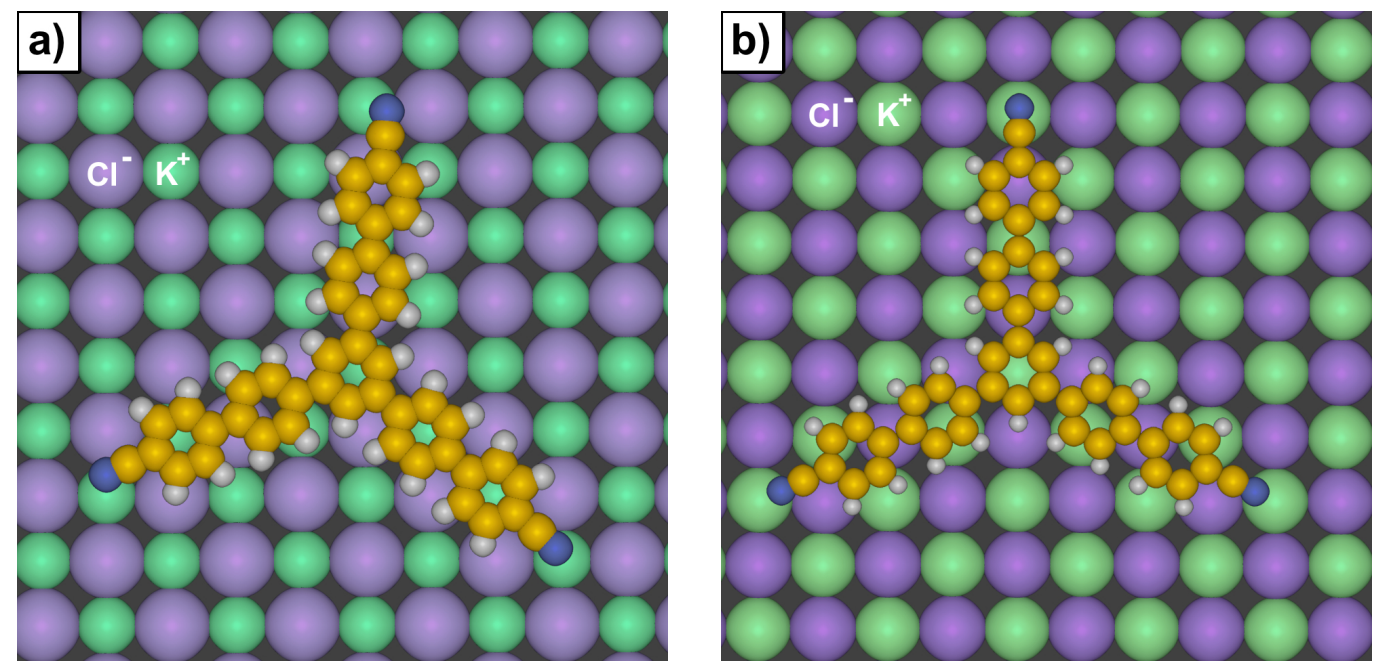

Figure 2: Two stable adsorption geometries of a single TCB molecule on a $\mathrm{KCl}(100)$ surface, calculated using the newly parameterized force field. The geometry (a) is the most stable with an adsorption energy of $4.55 \mathrm{eV}$ compared to the meta- stable state (b) with an adsorption energy of $4.3 \mathrm{eV}$.

by DFT calculations and the adsorption geometries are reproduced well.

In configuration Figure 2(b) the molecule exhibits mirror symmetry along the $\langle 100\rangle$ direction of the $\mathrm{KCl}$ surface. The adsorption energy of this configuration was found to be $4.3 \mathrm{eV}$. The molecule adapts a slightly deformed geometry, as the angles between the legs need to stretch and bend to fit to the optimum geometry. The benzene rings of the leg aligned along $\langle 100\rangle$ direction are flat, while the two benzene rings of the other two legs tilt by $9^{\circ}$ in opposite directions.

Adsorption configurations of the $\mathrm{CDB}$ molecule on $\mathrm{KCl}(001)$ have been discussed in detail in refs. ${ }^{39,40}$ The molecule attaches with the two CN groups and one of the two $\mathrm{O}$ atoms (see Figure 1) to K cations on the surface.

\section{Dynamic Adsorption Energy}

At non-zero temperatures the adsorption energies are reduced by entropic contributions. In this study we investigated the effect of molecular flexibility on the adsorption of organic molecules on an insulating surface by comparing the entropic contributions to adsorption of 


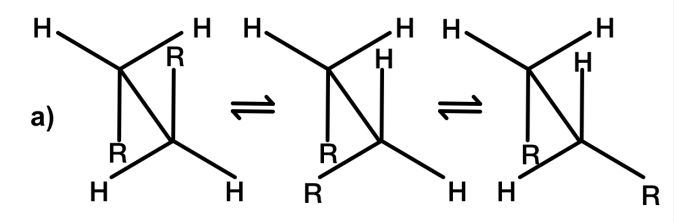

b)

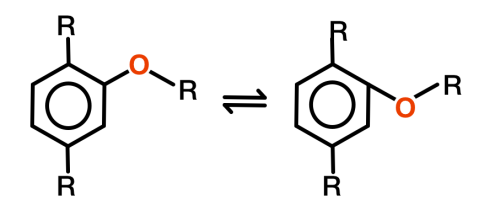

c)

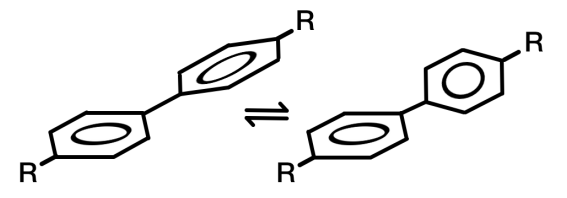

Figure 3: Different rotamers present in the system and their commensurate configurations, for a) a single bond in a linear carbonyl chain, b) the benzene-oxygen bond in CDB and c) the benzene-benzene bond.

a rigid TCB molecule with a more flexible CDB molecule.

\section{harmm}

The flexibility of these molecules can be described in terms of conformational entropy. Assuming that there are $\mathrm{N}$ different conformations of a molecule each with probability $p_{i}$, then the conformational entropy with reference to a rigid state is given as:

$$
\Delta S_{\text {conf }}=-R \sum_{i=1}^{N} p_{i} \ln \left(p_{i}\right)
$$

In order to evaluate conformational entropy of a molecule, it is useful to begin by defining the number of conformations in terms of populated rotamers. A rotamer is a conformational isomerism, where different conformations can be interconverted by rotating single bonds. $\mathrm{CDB}$ has four kinds of rotamers; the $\mathrm{C}-\mathrm{C}$ bond in the carbonyl chains, the C'-C' bond between the benzene rings the $\mathrm{C}-\mathrm{O}$ bond of the carbonyl chain and the phenyl-oxygen bond (C'-O), while TCB has only C'-C' rotamers between the phenyl groups, where C' indicates a carbon atom that belongs to a benzene ring.

A C-C bond of the carbonyl chains has three different conformations (see Figure 3). As- 
suming that these conformations are all equally probable, the conformational entropy per $\mathrm{C}-\mathrm{C}$ bond is $9.5 \times 10^{-5} \mathrm{eV} / \mathrm{K}$. The C'-C' bond of the phenyl groups has two conformations, since the torsional angle between the two benzene rings can be $\pm 31^{\circ}$ and thus the conformational entropy amounts to $0.36 \times 10^{-3} \mathrm{eV} / \mathrm{K}$. The $\mathrm{C}-\mathrm{O}$ bond has two conformations as well, one where the C'-O-C angle is pointing up out of the benzene plane and one where it is pointing down. Hence the conformational entropy per C'-O bond is the same as for a C'-C' bond. A C-O bond again has three possible conformations and hence the same entropy as a $\mathrm{C}-\mathrm{C}$ bond. Note the $\mathrm{C}-\mathrm{N} \equiv \mathrm{N}$ bonds are linear and freezing rotations about the bond axis does not result in a loss of conformations. Therefore they do not contribute to the conformational entropy calculation.

The CDB molecule contains 18 C-C bonds, two C-O bonds and 2 C'-C' bonds, compared to the TCB molecule which only has 6 C'-C' bonds. Summing up the corresponding energies at $300 \mathrm{~K}$, the conformational entropy amounts to $\Delta S_{\text {conf. }}^{C D B}=0.64 \mathrm{eV}$ and $\Delta S_{\text {conf. }}^{T C B}=0.11 \mathrm{eV}$. This difference in conformational entropy will be used as a metric to distinguish between flexible and rigid molecules.

\section{Molecular diffusion and rotation}

Using the optimized classical force fields, we simulated the dynamic processes that occur when $\mathrm{CDB}$ and TCB molecules are deposited onto the $\mathrm{KCl}(001)$ surface. The starting point for these studies was to examine the behavior of a single molecule on a perfect $\mathrm{KCl}(001)$ surface. These calculations were performed by placing a molecule above the $\mathrm{KCl}(001)$ surface with the plane of the molecule parallel to the surface and allowing the system to equilibrate for 10.0 ps. The simulations were performed using a 1.0 fs timestep and a 4 layer thick slab of $\mathrm{KCl}(001)$ to represent the surface. The bottom layer was fixed while the top 3 layers and all $\mathrm{TCB} / \mathrm{CDB}$ atoms were allowed to relax. The behavior of a single molecule on $\mathrm{KCl}(001)$ was

then observed for $10.0 \mathrm{~ns}$ at $300 \mathrm{~K}, 400 \mathrm{~K}$, and $500 \mathrm{~K}$. Simulations were repeated 10 times at each of these temperatures. 
The CDB molecule was observed to be mobile at room temperature and could easily reorient itself with respect to the $\mathrm{KCl}(001)$ surface. This mobility can be characterized in two ways. The first is by considering how often the molecule may rotate. Within the scope of this evaluation, we defined rotation as a change in the angle between the line drawn through the two cations on the surface that the $\mathrm{CN}$ groups of the molecule were closest to and the [100] direction of the surface. Throughout these simulations, the CDB molecule was observed to undergo an average of 5 rotational motions at $300 \mathrm{~K}$. At the same time, the central ring of the $\mathrm{CDB}$ molecule moved on average about $1.0 \mathrm{~nm}$ across the $\mathrm{KCl}(001)$ surface. At $400 \mathrm{~K}$, CDB molecules became predictably more mobile. Additionally, they were observed to occasionally roll or flip across the surface. Finally, at $500 \mathrm{~K}$ these effects were further pronounced, and in one rare instance the molecule became fully desorbed from the surface.

In comparison, molecular diffusion occurs at a much slower rate for TCB molecules. During $10.0 \mathrm{~ns}$ MD runs at $300 \mathrm{~K}$ the central benzene ring of the molecule stayed above the same cation site for the entire simulation or moved only to a neighboring cation $4.4 \AA$ away. However, the molecule is still able to rotate readily on the surface. For this system a rotational motion was defined as a change in which leg was aligned with a cation row. An average of 8 such rotations were observed within the $10.0 \mathrm{~ns}$ simulations. Note that these rotations are not directly comparable to those of the CDB molecule since they represent unique motions. At $400 \mathrm{~K}$ the molecule became more mobile and rotated rapidly. Occasionally one leg would lift up from the surface for less than 10.0 ps before going back to the planar adsorption geometry. At $500 \mathrm{~K}$ the dynamics became even faster, with very rapid rotation and the molecule diffusing over the surface with an average distance of $6.0 \mathrm{~nm}$ during one MD run.

\section{Temperature dependence of adsorption energy}

In order to investigate the physical meaning of adsorption energy at higher temperatures and to gain insight into entropic contributions, potential of mean force (PMF) calculations were 
Table 2: The equilibrium distance between a single TCB or CDB molecule and the surface and their adsorption energies are displayed for a range of temperatures.

\begin{tabular}{|c|ll|ll|}
\hline \multirow{2}{*}{ Temp. $(\mathrm{K})$} & \multicolumn{2}{|c|}{ TCB } & \multicolumn{2}{c|}{ CDB } \\
& Equilibrium & Adsorption & Equilibrium & Adsorption \\
& Distance $(\AA)$ & Energy $(\mathrm{eV})$ & Distance $(\AA)$ & Energy $(\mathrm{eV})$ \\
\hline \hline $0 \mathrm{~K}$ & 2.89 & -4.55 & 3.27 & -3.1 \\
$300 \mathrm{~K}$ & 3.16 & -3.95 & 3.39 & -2.37 \\
$400 \mathrm{~K}$ & 3.24 & -3.84 & 3.48 & -2.21 \\
$500 \mathrm{~K}$ & 3.33 & -3.74 & 3.65 & -2.06 \\
\hline
\end{tabular}

performed. It is important to note that the PMF must be defined in respect to a reference state, where the distance between the molecule and the surface is large enough for the force between the molecule and the surface to approach zero. This distance was determined to be $20.0 \AA$ for both molecules. The equilibrium distance between the molecule and the surface and the adsorption energy at each temperature can then be obtained from the minima of the calculated potential curves. The adsorption energies of CDB and TCB molecules as a function of temperature are shown in Table 2.

As the temperature increases from $0 \mathrm{~K}$ to room temperature and higher, the adsorption energies of the TCB and CDB molecules are reduced substantially. At room temperature the adsorption energy for a single TCB molecule is $0.6 \mathrm{eV}$ lower than the value obtained from the static calculations. This effect is even more pronounced for the CDB molecule, where the adsorption energy is $0.7 \mathrm{eV}$ lower at room temperature than at $0 \mathrm{~K}$. These results indicate that ignoring the temperature dependence of adsorption energy can potentially lead to false conclusions regarding the strength of adsorption. This change in adsorption energy can be attributed to entropy and requires further analysis.

\section{Entropy contribution to adsorption}

The entropic contribution to adsorption can be calculated using the PMF data. In Figure 4 the potential of mean force is plotted for the TCB and CDB molecule, respectively. The 
change in free energy of the system is given by

$$
G_{\text {adsorbed }}-G_{\text {desorbed }}=\Delta G=\Delta H-T \Delta S
$$

where the difference is taken between the adsorbed and the desorbed state. The minima in the PMF curves correspond to free energy minima, since entropy is included in the MD calculations. Therefore, assuming that $\Delta H$ and $\Delta S$ are constant over the temperature range considered, the gradient of a linear fit through the potential minima gives a rough estimate of the entropic contribution to adsorption $\Delta S .{ }^{62,64}$ At $300 \mathrm{~K}$ the entropic contribution to adsorption amounts to $0.4 \mathrm{eV}$ per TCB molecule on the $\mathrm{KCl}(001)$ surface. Since TCB is a large rigid molecule, the adsorption enthalpy is an order of magnitude larger than the entropy contribution to adsorption. In comparison, the linear fit results in a much larger entropic contribution for the flexible CDB molecule. At $300 \mathrm{~K}$ the change in entropy was calculated to be $0.7 \mathrm{eV}$. This larger entropic contribution can be attributed to the loss in conformational freedom. As the molecule adsorbs on the surface it is forced into a more planar geometry and hence cannot access all conformational states. Since TCB has fewer conformational states and is more rigid, the entropic contribution is smaller compared to CDB.

However, considering the change in enthalpy and the change in entropy to be constant
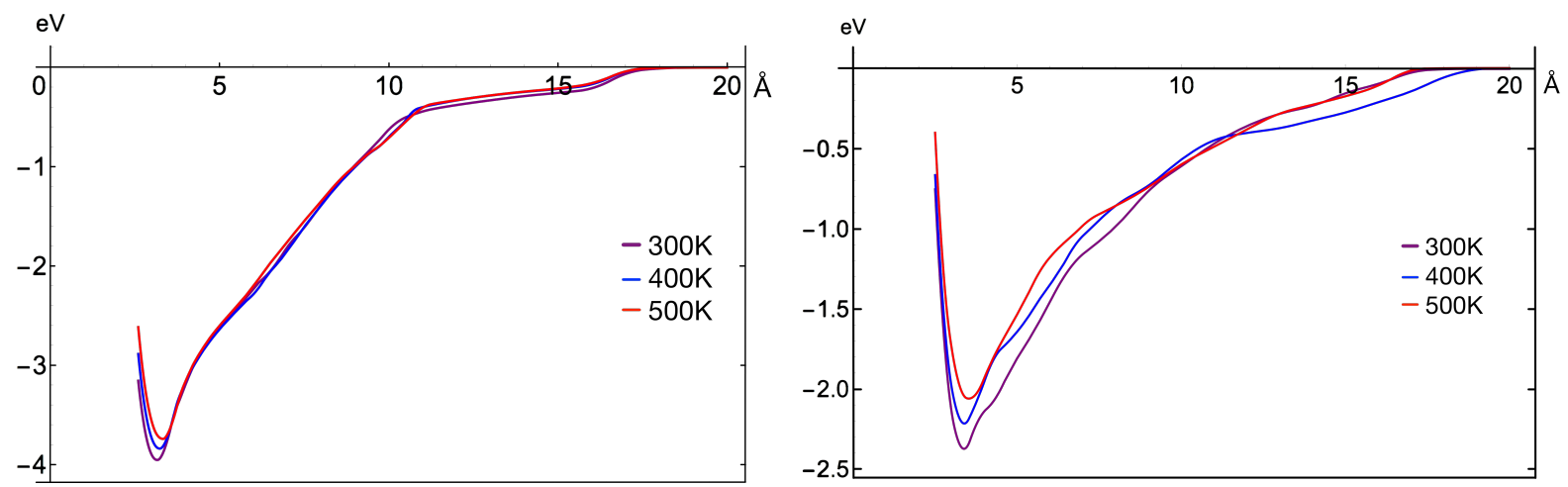

Figure 4: Potential of mean force curves for TCB (left) and CDB (right) on a $\mathrm{KCl}(001)$ substrate at $300 \mathrm{~K}, 400 \mathrm{~K}$ and $500 \mathrm{~K}$, respectively. The potentials were defined to be zero when the center of mass of the molecules reached a distance of $20 \AA$ away from the surface. 
over a temperature range may not be physically accurate. As the temperature changes, so do the dynamics of the system. At higher temperatures, different vibrational and rotational modes are accessible, resulting in a change in $\Delta S$. A more detailed method of calculating the entropy change is given by Smith et al. ${ }^{65}$ where the entropic component to the free energy is written as

$$
-T S(r)=-T S\left(r_{0}\right)+\frac{1}{k T} \int_{r_{0}}^{r} d R\left[\left\langle E \frac{\partial E}{\partial R}\right\rangle_{R}-\langle E\rangle_{R}\left\langle\frac{\partial E}{\partial R}\right\rangle_{R}\right] .
$$

Here $E$ represents the total potential energy and $\langle\ldots\rangle_{R}$ is the ensemble average when the distance between the molecule's center of mass and the surface is fixed at $R$. The additive constant $T S\left(r_{0}\right)$ is chosen such that $-T S(r)$ is zero at $r=20.0 \AA$, at which distance the molecule is completely desorbed from the surface. Therefore Eq. 6 gives the change in entropy as a function of the molecule - surface separation. A plot of this change in entropy is given in Figure 5 for TCB at $300 \mathrm{~K}, 400 \mathrm{~K}$ and $500 \mathrm{~K}$, respectively.

The entropy change displays three stepwise decreases as the TCB molecule adsorbs onto the $\mathrm{KCl}$ (001) surface. As the molecule approaches the surface, one of the legs will attach to the surface and the molecule will lose some translational and rotational entropy. This initial change in entropy is illustrated in Figure 5 at position (c). Since the center of mass is fixed in these calculations, initially the molecule's motion becomes trapped in this position and it is unable to translate across the surface, leading to a large decrease in entropy. The entropy of the system increases again when the COM is brought nearer to the surface as the molecule can reorient itself, which allows for more molecular motion. A second drop in entropy is observed when another leg attaches to the surface (see Figure 5(b)) and the entropy continues to decrease until the optimum adsorption geometry is reached in the configuration shown in Figure 5(a). Forcing the molecule even closer to the surface will lead to a huge decrease in entropy, as the molecular motion becomes frozen and its degrees of freedom become fixed.

A similar behavior can be observed for the CDB molecule with some key differences (see 

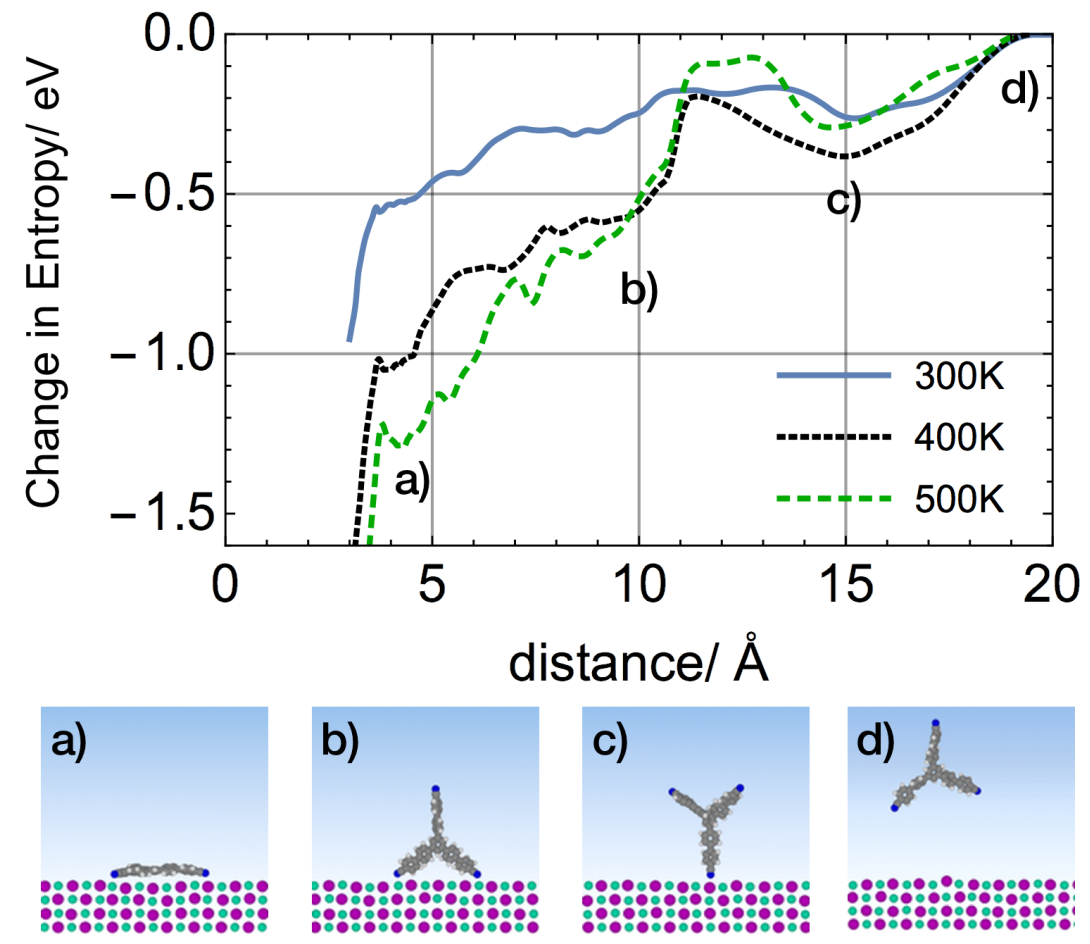

Figure 5: Change in entropy plotted against distance from surface for a TCB molecule. The curves represent a system at $300 \mathrm{~K}, 400 \mathrm{~K}$ and $500 \mathrm{~K}$, respectively. Below the graph are snapshots of TCB at various stages in the adsorption, as indicated in the plot.

Figure 6). First, the entropy decreases more smoothly at all three temperatures. This can be attributed to the fact that CDB is more flexible and has many more degrees of freedom. As the molecule adsorbs on the surface, these degrees of freedom are gradually restricted, leading to a smoother decrease in entropy. Secondly the magnitude of the change in entropy upon adsorption is much larger, increasing form $T \Delta S=1.0 \mathrm{eV}$ at $300 \mathrm{~K}$ to more than $5.0 \mathrm{eV}$ at $500 \mathrm{~K}$. At $400 \mathrm{~K}$ the adsorption enthalpy is already similar to the change in entropy and the molecule is expected to desorb from the surface. Movies showing the adsorption of TCB and $\mathrm{CDB}$ in more detail can be found in the supplementary information.

The large changes in entropy observed for both rigid TCB and flexible CDB molecules show that these effects cannot be neglected at elevated temperatures. Comparing the two methods of estimating the change in entropy upon adsorption, it becomes clear that the assumption of $\Delta S$ being a constant over the temperature range considered leads to an un- 


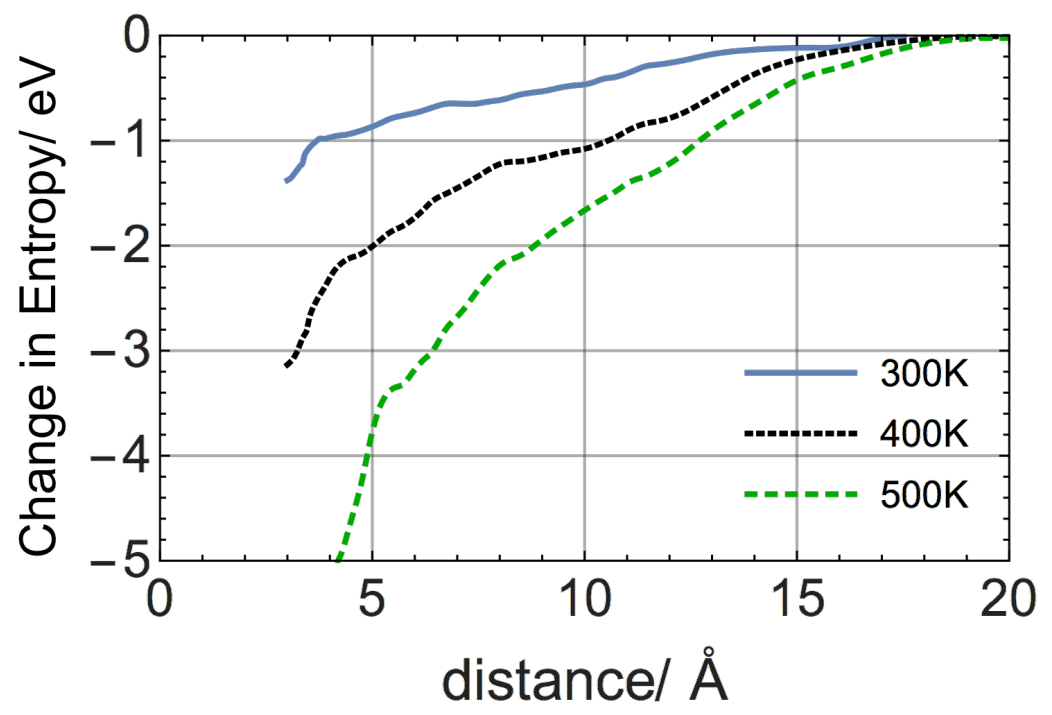

Figure 6: Change in entropy plotted against distance for the CDB molecule at temperatures of $300 \mathrm{~K}, 400 \mathrm{~K}$ and $500 \mathrm{~K}$, respectively.

derestimation at higher temperatures, whereas the method proposed by Smith et al. ${ }^{65}$ gives a much more detailed understanding of the change in entropy upon adsorption.

\section{Conclusions}

Our comparative study of adsorption of two organic molecules on the $\mathrm{KCl}$ (001) surface revealed two main results. Firstly, using DFT-D3 overestimates the vdW contribution to the adsorption enthalpy of molecules of this type by only about $20 \%$ and is therefore a computationally efficient way of studying their adsorption. Secondly, entropy losses upon adsorption are significant for flexible molecules; at high enough temperatures they can match the enthalpic contribution to adsorption energy.

A thorough understanding of dynamics of organic molecules at insulating surfaces is imperative for the development of molecular devices. The adsorption energy and geometry are dependent on both the character of interactions and temperature. As a molecule adsorbs on a surface, some of its degrees of freedom become constrained, leading to the entropy loss. For example, we found that for the relatively rigid TCB molecule the entropy loss does not 
exceed the adsorption enthalpy up to $500 \mathrm{~K}$. However, the flexible CDB molecule exhibits an entropy loss comparable to the adsorption energy already below $400 \mathrm{~K}$ and can desorb from the surface.

These results complement static calculations of molecular adsorption and shed further light on the dynamic behavior of molecules at surfaces. They can be useful for our understanding of expected stability of molecular structures and the mechanisms of self-assembly.

\section{Acknowledgement}

JG is supported by EPSRC and JAIST. The authors acknowledge the use of the ARCHER high-performance computing facilities via our membership to the UK's HPC Materials Chemistry Consortium, which is funded by EPSRC (EP/L000202). DG acknowledges the use of the Serenity computing cluster, and support services provided by Nanolayers Research Computing for the completion of this work. The authors would also like to thank F. Federici Canova for his help in parameterizing the force field for TCB.

\section{References}

(1) Mason, A.; Mukhopadhyay, S. C.; Jayasundera, K. P. Sensing Technology: Current Status and Future Trends III; Springer: New York City, USA, 2015.

(2) Chaki, N. K.; Vijayamohanan, K. Self-assembled monolayers as a tunable platform for biosensor applications. Biosens. Bioelectron. 2002, 17, 1-12.

(3) Maboudian, R.; Ashurst, W.; Carraro, C. Self-assembled monolayers as anti-stiction coatings for MEMS: characteristics and recent developments. Sensor Actuat. A-Phys. 2000, 82, $219-223$.

(4) Besenbacher, F.; Lauritsen, J. V.; Wendt, S. STM studies of model catalysts. Nano Today 2007, 2, 30 - 39. 
(5) Zhang, X.; Shen, J. Self-Assembled Ultrathin Films: From Layered Nano-architectures to Functional Assemblies. Adv. Mater. 1999, 11, 1139-1143.

(6) Joachim, C.; Gimezewski, J.; Aviram, A. Electronics using hybrid-molecular and monomolecular devices. Nature 2000, 408, 541-548.

(7) Heath, J. R. Molecular electronics. Annu. Rev. Mater. Sci. 2009, 39, 1-23.

(8) Song, H.; Reed, M. A.; Lee, T. Single molecule electronic devices. Adv. Mater. 2011, 23, $1583-1608$.

(9) Neff, J. L.; Kittelmann, M.; Bechstein, R.; Kühnle, A. Decisive influence of substitution positions in molecular self-assembly. PCCP 2014, 16, 15437.

(10) Hinault, A.; Pujol, A.; Chaumeton, F.; Martrou, D.; Gourdon, A.; Gauthier, S. An NC-AFM and KPFM study of the adsorption of a triphenylene derivate on $\mathrm{KBr}(001)$. Beilstein J. Nanotechnol. 2012, 3, 221.

(11) Hauke, C. M.; Bechstein, R.; Kittelmann, M.; Storz, C.; Kilbinger, A. F. M.; Rahe, P.; Kühnle, A. Controlling molecular self-assembly on an insulating surface by rationally designing an efficient anchor functionality that maintains structural flexibility. ACS Nano 2013, 7, 5491-5498.

(12) Pawlak, R.; Nony, L.; Bocquet, F.; Oison, V.; Sassi, M.; Debierre, J. M.; Loppacher, C.; Porte, L. Supramolecular assemblies of 1,4-benzene diboronic acid on $\mathrm{KCl}(001)$. J. Phys. Chem. C 2010, 114, 9290-9295.

(13) Rahe, P.; Nimmrich, M.; Kühnle, A. Templating: substrate templating upon selfassembly of hydrogen-bonded molecular networks on an insulating surface. Small 2012, 8, 2968-2968.

(14) Loppacher, C.; Zerweck, U.; Eng, L. M.; Gemming, S.; Seifert, G.; Olbrich, C.; 
Morawetz, K.; Schreiber, M. Adsorption of PTCDA on a partially KBr covered Ag(111) substrate. Nanotechnology 2006, 17, 1568-1573.

(15) Hentschke, R.; Schtirmann, B. L.; Rabe, J. P. Molecular Dynamics Simulations of Ordered Alkane Chains Physisorbed on Graphite. J. Chem. Phys. 1992, 96, 62136221.

(16) Kustmann, T.; Schlarb, A.; Fendrich, M.; Wagner, T.; Möller, R.; Hoffmann, R. Dynamic force microscopy study of 3,4,9,10-perylenetetracarboxylic dianhydride on $\operatorname{KBr}(001)$. Phys. Rev. 2005, 71, 121403(R).

(17) Sasahara, A.; Uetsuka, H.; Ishibashi, T.; Onishi, H. A needle-like organic molecule imaged by noncontact atomic force microscopy. Appl. Surf. Sci. 2002, 188, 265-271.

(18) Namai, Y.; Fukui, K.; Iwasawa, Y. The dynamic behaviour of CH3OH and NO2 adsorbed on CeO2(111) studied by noncontact atomic force microscopy. Nanotechnology 2004, 15, S49-S54.

(19) Nony, L.; Bennewitz, R.; Pfeiffer, O.; Gnecco, E.; Baratoff, A.; Meyer, E.; Eguchi, T.; Gourdon, A.; Joachim, C. Cu-TBPP and PTCDA molecules on insulating surfaces studied by ultra-high-vacuum non-contact AFM. Nanotechnology 2004, 15, S91-S96.

(20) Burke, S. A.; Mativetsky, J. M.; Hoffmann, R.; Grütter, P. Nucleation and submonolayer growth of C60 on KBr. Phys. Rev. Lett. 2005, 94, 096102(1-4).

(21) Dienel, T.; Loppacher, C.; Mannsfeld, S. C. B.; Forker, R.; Fritz, T. Growth-modeinduced narrowing of optical spectra of an organic adlayer. Adv. Mater. 2008, 20, 959-963.

(22) Burke, S. A.; Ji, W.; Mativetsky, J. M.; Topple, J. M.; Fostner, S.; Gao, H.-J.; Guo, H.; Grütter, P. Strain induced dewetting of a molecular system: Bimodal growth of PTCDA on NaCl. Phys. Rev. Lett. 2008, 100, 186104. 
(23) Schütte, J.; Bechstein, R.; Rahe, P.; Rohlfing, M.; Kühnle, A.; Langhals, H. Imaging perylene derivatives on rutile $\mathrm{TiO}_{2}(110)$ by noncontact atomic force microscopy. Phys. Rev. B 2009, 79, 045428.

(24) Loske, F.; Bechstein, R.; Schütte, J.; Ostendorf, F.; Reichling, M.; Kühnle, A. Growth of ordered C60 islands on TiO2(110). Nanotechnology 2009, 20, 065606.

(25) Kittelmann, M.; Rahe, P.; Kühnle, A. Molecular self-assembly on an insulating surface: interplay between substrate templating and intermolecular interactions. J. Phys.: Condens. Matter 2012, 24, 354007.

(26) Lämmle, K.; Trevethan, T.; Schwarz, A.; Watkins, M.; Shluger, A.; Wiesendanger, R. Unambiguous determination of the adsorption geometry of a metal-organic complex on a bulk insulator. Nano Lett. 2010, 10, 2965-2971.

(27) Schwarz, A.; Gao, D. Z.; Lämmle, K.; Grenz, J.; Watkins, M. B.; Shluger, A. L.; Wiesendanger, R. Determining adsorption geometry, bonding, and translational pathways of a metal-organic complex on an oxide surface: Co-Salen on $\mathrm{NiO}(001)$. J. Phys. Chem. C 2013, 117, 1105-1112.

(28) Iwata, K.; Yamazaki, S.; Mutombo, P.; Hapala, P.; Ondracek, M.; Jelinek, P.; Sugimoto, Y. Chemical structure imaging of a single molecule by atomic force microscopy at room temperature. Nat. Commun. 2015, 6, 1-7.

(29) Gao, D. Z.; Grenz, J.; Watkins, M. B.; Federici Canova, F.; Schwarz, A.; Wiesendanger, R.; Shluger, A. L. Using metallic noncontact atomic force microscope tips for imaging insulators and polar molecules: tip characterization and imaging mechanisms. ACS nano 2014, 8, 5339-51.

(30) Zhang, J.; Chen, P.; Yuan, B.; Ji, W.; Cheng, Z.; Qiu, X. Real-space identification of intermolecular bonding with atomic force microscopy. Science 2013, 342, 611-614. 
(31) Hämäläinen, S. K.; van der Heijden, N.; van der Lit, J.; den Hartog, S.; Liljeroth, P.; Swart, I. Intermolecular contrast in atomic force microscopy images without intermolecular bonds. Phys. Rev. Lett. 2014, 113, 186102.

(32) Barth, C.; Gingras, M.; Foster, A. S.; Gulans, A.; Félix, G.; Hynninen, T.; Peresutti, R.; Henry, C. R. Two-dimensional nanostructured growth of nanoclusters and molecules on insulating surfaces. Adv. Mater. 2012, 24, 3228-32.

(33) Kemball, C. Entropy of Adsorption, in Advances in Catalysis and Related Subjects, vol. II; Academic Press: New York City, USA, 1950.

(34) Ben Tal, N.; Honig, B.; Bagdassarian, C. K.; Ben Shaul, A. Association entropy in adsorption processes. Biophys. J. 2000, 79, 1180-7.

(35) Freeman, C. L.; Harding, J. H. Entropy of Molecular Binding at Solvated Mineral Surfaces. J. Phys. Chem. C 2013, 118, 1506-1514.

(36) Alfè, D.; Gillan, M. J. Ab initio statistical mechanics of surface adsorption and desorption. J. Chem. Phys. 2007, 127, 114709.

(37) Campbell, C. T.; Sellers, J. R. V. Enthalpies and entropies of adsorption on well-defined oxide surfaces: Experimental measurements. Chem. Rev. 2013, 113, 4106-4135.

(38) Rossel, F.; Pivetta, M.; Patthey, F.; Ćavar, E.; Seitsonen, A. P.; Schneider, W.-D. Growth and characterization of fullerene nanocrystals on $\mathrm{NaCl} / \mathrm{Au}(111)$. Physical Review $B$ 2011, 84, 075426.

(39) Amrous, A.; Bocquet, F.; Nony, L.; Para, F.; Loppacher, C.; Lamare, S.; Palmino, F.; Cherioux, F.; Gao, D. Z.; Canova, F. F. et al. Molecular design and control over the morphology of self-assembled films on ionic substrates. Adv. Mater. Interf. 2014, 1, 1400414 . 
(40) Gao, D. Z.; Federici Canova, F.; Watkins, M. B.; Shluger, A. L. Efficient parametrization of complex molecule-surface force fields. J. Comput. Chem. 2015, 36, 1187-1195.

(41) Laino, T.; Mohamed, F.; Laio, A.; Parrinello, M. An Efficient Real Space Multigrid QM/MM Electrostatic Coupling. J. Chem. Theory Comput. 2005, 1, 1176-1184.

(42) Laino, T.; Mohamed, F.; Laio, A.; Parrinello, M. An Efficient Linear-Scaling Electrostatic Coupling for Treating Periodic Boundary Conditions in QM/MM Simulations. J. Chem. Theory Comput. 2006, 2, 1370-1378.

(43) VandeVondele, J.; Krack, M.; Mohamed, F.; Parrinello, M.; Chassaing, T.; Hutter, J. Quickstep: Fast and accurate density functional calculations using a mixed Gaussian and plane waves approach. Comp. Phys. Commun. 2005, 167, 103-128.

(44) Perdew, J. P.; Burke, K.; Ernzerhof, M. Generalized Gradient Approximation Made Simple. Phys. Rev. Lett. 1996, 77, 3865-3868.

(45) Grimme, S. Semiempirical GGA-type density functional constructed with a long-range dispersion correction. J. Comput. Chem. 2006, 27, 1787-1799.

(46) VandeVondele, J.; Hutter, J. Gaussian basis sets for accurate calculations on molecular systems in gas and condensed phases. J. Chem. Phys. 2007, 127, 114105-1-9.

(47) Vogt, J.; Weiss, H. The structure of $\mathrm{NaCl}\left(\begin{array}{lll}1 & 0 & 0\end{array}\right)$ and $\mathrm{KCl}\left(\begin{array}{lll}1 & 0 & 0\end{array}\right)$ single crystal surfaces: a tensor low energy electron diffraction analysis. Surf. Sci. 2001, 491, 155-168.

(48) Del Ben, M.; Hutter, J.; VandeVondele, J. Second-order Møller-Plesset perturbation theory in the condensed phase: an efficient and massively parallel Gaussian and plane waves approach. J. Chem. Theory Comput. 2012, 8, 4177-4188.

(49) Del Ben, M.; Hutter, J.; VandeVondele, J. Forces and stress in second order MøllerPlesset perturbation theory for condensed phase systems within the resolution-ofidentity Gaussian and plane waves approach. J. Chem. Phys. 2015, 143, 102803. 
(50) Del Ben, M.; Hutter, J.; VandeVondele, J. Electron correlation in the condensed phase from a resolution of identity approach based on the Gaussian and plane waves scheme. J. Chem. Theory Comput. 2013, 9, 2654-2671.

(51) Gœdecker, S.; Teter, M.; Hutter, J. Separable dual-space Gaussian pseudopotentials. Phys. Rev. B 1996, 54, 1703.

(52) Hartwigsen, C.; Gœedecker, S.; Hutter, J. Relativistic separable dual-space Gaussian pseudopotentials from H to Rn. Phys. Rev. B 1998, 58, 3641.

(53) Eichkorn, K.; Treutler, O.; Öhm, H.; Häser, M.; Ahlrichs, R. Auxiliary basis sets to approximate Coulomb potentials. Chemical physics letters 1995, 240, 283-290.

(54) Feyereisen, M.; Fitzgerald, G.; Komornicki, A. Use of approximate integrals in ab initio theory. An application in MP2 energy calculations. Chemical Physics Letters 1993, 208, 359-363.

(55) Zhao, Y.; ; Truhlar, D. G. Infinite-basis calculations of binding energies for the hydrogen bonded and stacked tetramers of formic acid and formamide and their use for validation of hybrid DFT and ab initio methods. J. Phys. Chem. A 2005, 109, 6624-6627.

(56) Brooks, B.; Bruccoleri, R.; Olafson, D.; States, D.; Swaminathan, S.; Karplus, M. CHARMM: A Program for Macromolecular Energy, Minimization, and Dynamics Calculations. Journal of Computational Chemistry 1983, 4, 187-217.

(57) Catlow, C. R. A.; Diller, K. M.; Norgett, M. J. Interionic potentials for alkali halides. Journal of Physics C: Solid State Physics 1977, 10, 1395-1412.

(58) Laino, T.; Mohamed, F.; Laio, A.; Parrinello, M. An efficient real space multigrid QM/MM electrostatic coupling. J. Chem. Theory Comput. 2005, 1, 1176-1184.

(59) Laino, T.; Mohamed, F.; Laio, A.; Parrinello, M. An efficient linear-scaling electrostatic 
coupling for treating periodic boundary conditions in QM/MM simulations. J. Chem. Theory Comput. 2006, 2, 1370-1378.

(60) Doudou, S.; Burton, N. A.; Henchman, R. H. Standard free energy of binding from a one-dimensional potential of mean force. J. Chem. Theory Comput. 2009, 5, 909-918.

(61) Roux, B. The calculation of the potential of mean force using computer simulations. Comput. Phys. Commun. 1995, 91, 275-282.

(62) Fox, H.; Gillan, M.; Horsfield, A. Methods for calculating the desorption rate of an isolated molecule from a surface: Water on $\mathrm{MgO}(001)$. Surface Science 2007, 601, $5016-5025$.

(63) Hunter, C. A.; Sanders, J. K. M. The nature of pi-pi interactions. JACS 1990, 112, $5525-5534$.

(64) Alfè, D.; Gillan, M. J. Absolute rate of thermal desorption from first-principles simulation. J. Phys. Condens. Matter 2006, 18, L451-7.

(65) Smith, D. E.; Zhang, L.; Haymet, A. D. J. Entropy of association of methane in water: a new molecular dynamics computer simulation. J. Am. Chem. Soc. 1992, 114, 58755876 . 